\title{
AIAA 93-0192 \\ A COMPARISON OF THE PREDICTIVE CAPABILITIES OF SEVERAL TURBULENCE MODELS USING UPWIND AND CENTRAL-DIFFERENCE COMPUTER CODES
}

\author{
Christopher L. Rumsey* and Veer N. Vatsa* \\ NASA Langley Research Center \\ Hampton, Virginia 23681-0001
}

\begin{abstract}
Four turbulence models are described and evaluated for transonic flows using the upwind code CFL $B D$ and the central-difference code TLNS3D. In particular, the effects of recent modifications to the half-equation model of Johnson-King are explored in detail, and different versions of the model are compared. This model can obtain good results for both two-dimensional (2-D) and three-dimensional (3-D) separated flows. The one-equation models of Baldwin-Barth and Spalart-Allmaras perform well for separated airfoil flows, but can predict the shock too far forward at the outboard stations of a separated wing. The equilibrium model of Baldwin-Lomax predicts the shock location too far aft for both 2-D and 3-D separated flows, as expected. In general, all models perform well for attached or mildly separated flows.
\end{abstract}

\section{Introduction}

As computational fluid dynamics (CFD) grows in capability as a tool for the analysis of three-dimensional (3-D) aerospace configurations, turbulence modeling continues to be one of the primary factors that inhibits more widespread usage of Navier-Stokes codes by aircraft manufacturers. The search for a model that accurately predicts both attached and separated 3-D flow fields is complicated by the fact that it is difficult to assess the capabilities of new or refined turbulence models because of inherent limitations in the CFD codes that use them. Particularly relevant is the issue of truncation error; the density of the grid used, the type of differencing scheme employed, and, for central-difference schemes, the amount of artificial dissipation added for numerical stability are all likely to have an effect on the solution.

Turbulence models are often assessed based on a limited study - generally only one or two cases

\footnotetext{
* Fluid Mechanics Division, Member AIAA

Copyright $@ 1993$ by the American Institute of Aeronautics and Astronautics, Inc. No copyright is asserted in the United States under Title 17, U.S. Code. The U.S. Government has a royalty-free license to exercise all rights under the copyright claimed herein for Governmental purposes. All other rights are reserved by the copyright owner.
}

are analyzed using a single computer code with little or no variation in grid density. As will be shown in this paper, such computed results can sometimes agree with experimental data almost coincidentally; reducing the truncation error through mesh refinement or lowering the dissipation levels can sometimes lead to dramatically different results, particularly for 3-D separated flows. By conducting a broader study that covers both two- and three-dimensional configurations and using grid refinement studies with more than one computer code, the effects of truncation error can be determined. Hence, a more accurate assessment of the turbulence models is possible.

This paper investigates some of these issues for the studies of transonic flow over the ONERA M6 wing [1] and the Lockheed Wing C [2]. Two widely used computer codes, CFL3D [3] and TLNS3D [4], are employed. Each of these codes can employ either the Baldwin-Lomax [5] or the Johnson-King [6] turbulence model. Additionally, CFL3D can employ the BaldwinBarth [7] or the Spalart-Allmaras [8] turbulence model. To provide a wider basis for assessment, the capabilities of the various turbulence models are examined in two dimensions using the two-dimensional (2-D) mode of CFL3D as well. 


\section{Method}

\subsection{The Computer Codes}

CFL3D and TLNS3D both solve the 3-D timedependent thin-layer Navier-Stokes equations with a finite-volume formulation. Both can employ grid sequencing, multigrid, and local time-stepping to accelerate convergence to steady state. When converged temporally to a steady-state solution, both methods are globally second-order accurate.

CFL3D, described in detail in [3], is an upwind code. For all results presented in this paper, upwindbiased spatial differencing is used for the inviscid terms, and flux limiting is used to obtain smooth solutions in the vicinity of shock waves. All viscous terms are centrally differenced. The equations are solved implicitly with the use of 3-factor approximate factorization (AF). Either Roe's flux difference splitting (FDS) [9] or van Leer's flux vector splitting (FVS) [10] can be employed to obtain fluxes at the cell faces.

TLNS3D, described in detail in [4], is a centraldifference code. Second-order central differences are used for all spatial derivatives, and a blend of seconddifference and fourth-difference artificial dissipation terms is used to maintain numerical stability. These artificial dissipation terms can be added in either scalar or matrix form [11]. The solution is advanced explicitly in time using either a four-stage or five-stage RungeKutta time-marching algorithm.

\subsection{The Turbulence Models}

Baldwin-Lomax. The Baldwin-Lomax (B-L) turbulence model [5] is used widely throughout the CFD community; its capabilities and limitations are wellknown. In short, it is generally considered a good model for the prediction of attached flows, but it is deficient for flows with any significant separated regions. In particular, the B-L model tends to predict shocks too far downstream for separated transonic flows over aerodynamic configurations.

The $\mathrm{B}-\mathrm{L}$ model is an algebraic model. The inner eddy viscosity is determined via

$$
\mu_{t, i_{(\mathrm{B}-\mathrm{L})}}=\rho\left(0.4 D_{(\mathrm{B}-\mathrm{L})} y\right)^{2} \Omega
$$

where

$$
D_{(\mathrm{B}-\mathrm{L})}=1-\exp \left(-y^{+} / 26\right)
$$

and

$$
y^{+}=y \sqrt{\rho_{w} \tau_{w}} / \mu_{w}
$$

where $y$ is the distance to the wall or wake cut, $\Omega$ is the magnitude of the vorticity, and the subscript $w$ refers to a wall value.

The outer eddy viscosity is given by

$$
\mu_{t, o_{(\mathrm{B}-\mathrm{L})}}=(0.0168)(1.6) \rho F_{\text {wake }} \Gamma_{(\mathrm{B}-\mathrm{L})}
$$

where

$$
F_{\text {wake }}=\min \left[y_{m} F_{m}, y_{m}\left(q_{\max }-q_{\min }\right)^{2} / F_{m}\right]
$$

and

$$
\Gamma_{(\mathrm{B}-\mathrm{L})}=1 /\left[1+5.5\left(0.3 y / y_{m}\right)^{6}\right]
$$

and

$$
F=y \Omega D_{(\mathrm{B}-\mathrm{L})}
$$

In wakes, $D_{(\mathrm{B}-\mathrm{L})}$ is taken as 1 . The subscript $m$ in equations (5) and (6) refers to the value at the location where $F$ is at its maximum along grid lines that are oriented normal to the body (i.e., in the viscous direction for thin-layer Navier-Stokes). The symbol $q$ denotes the total velocity.

The eddy viscosity $\mu_{t}$ is determined by marching away from the wall along these same grid lines and is taken as $\mu_{t, i}(\mathrm{~B}-\mathrm{L})$ from the wall (where the value is zero) to the point above the wall where $\mu_{t, i_{(\mathrm{B}-\mathrm{L})}}$ first exceeds $\mu_{t, o_{(\mathrm{B}-\mathrm{L})}}$. Thereafter $\mu_{t}$ is given the value $\mu_{t, o_{(\mathrm{B}-\mathrm{L})}}$. Transition is modeled by setting $\mu_{t}$ to zero along all grid lines that are normal to the wall within a preselected range.

Johnson-King. The Johnson-King (J-K) turbulence model is a so-called one-half equation turbulence model that requires the solution of an ordinary differential equation (ODE) for the maximum Reynolds shear stress over the body surface. Since its introduction [6], several modifications and enhancements have been made [12], [13], [14], [15]. An attempt will be made to describe the different versions of the model, as well as the effects of each of the individual modifications.

In one of the original versions of the J-K model [12], (termed J-K1985), the inner and outer eddy viscosities are given by

$$
\mu_{t, i_{(\mathrm{J}-\mathrm{K})}}=0.4 \rho\left(D_{(\mathrm{J}-\mathrm{K})}\right)^{2} y u_{m}
$$

and

$$
\mu_{t, o_{(\mathrm{J}-\mathrm{K})}}=0.0168 \rho q_{\delta} \delta^{*} \Gamma_{(\mathrm{J}-\mathrm{K})} \sigma
$$

where

$$
D_{(\mathrm{J}-\mathrm{K})}=1-\exp \left[-\rho_{w} y u_{T} /\left(A^{+} \mu_{w}\right)\right]
$$


and

$$
\Gamma_{(\mathrm{J}-\mathrm{K})}=1 /\left[1+5.5(y / \delta)^{6}\right]
$$

and $u_{m}$ and $u_{T}$ are velocity scales which are both taken to be the square root of the maximum Reynolds shear stress $(\overline{-u} m)^{2}$ along each grid line that is normal to the body. The displacement thickness $\delta^{*}$ is defined by

$$
\delta^{*}=\left(1-q / q_{\delta}\right)
$$

where $\delta$ represents the boundary-layer edge. $A^{+}$is taken to be 15 .

The term $\sigma$ is the modeling parameter that provides the link between the eddy-viscosity distribution and the rate equation for the development of the maximum Reynolds shear stress. The rate equation is given by

$$
\begin{aligned}
& \frac{D(\overline{\overline{-u}} \mathrm{~m})}{D}=\frac{0.25}{m}\left(\overline{\overline{-u}_{\mathrm{u}}}\right) \\
& {\left[(\overline{-u} m, \quad)^{2}-(\overline{-u} m)^{2}\right]-0.25 D_{m}}
\end{aligned}
$$

The turbulent diffusion term $D_{m}$ is given by

$$
D_{m}=\frac{0.5(\overline{-} m)^{2}\left(\sigma^{2}-1\right)}{0.25\left(0 . \delta-y_{m}\right)}
$$

and $m$ is the dissipation length scale

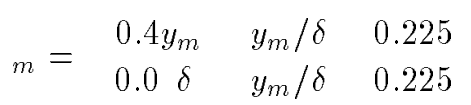

In equation (13), the subscript $e q$ denotes the equilibrium value of the maximum Reynolds shear stress. In equations (14) and (15), $y_{m}$ denotes the distance from the body to the location where $\overline{-u}$ is maximum.

As discussed in references [6] and [13], the ODE (13) can be linearized and solved for , where is defined as

$$
(\overline{-u \quad m})^{-2}
$$

The resulting value of is then used to update $\sigma$ through an iteration process. The inner and outer models are blended to obtain the eddy viscosity $\mu_{t}$ using

$$
\mu_{t}=\mu_{t, o(\mathrm{~J}-\mathrm{K})} 1-\exp -\frac{\mu_{t, i_{(\mathrm{J}-\mathrm{K})}}}{\mu_{t, o_{(\mathrm{J}-\mathrm{K})}}}
$$

J-K1985 does well in the computation of 2-D separated-flow airfoil cases [16], but tends to predict shocks too far forward for transonic attached equilibrium flows. Another disadvantage of this version of the model is that the determination of the boundary-layer edge $\delta$ is computationally difficult and rarely foolproof. In fact, this problem is considered by many to be a primary disadvantage of the Clauser type of outer eddyviscosity formulation and is one of the reasons why the B-L model was developed [5].

Abid et al. [13] extended the J-K model to 3-D flows. Their version (termed J-K1990A) uses equation (8) for the inner model, but the velocity scale $u_{T}$ in equation (10) is given by

$$
u_{T}=\mathrm{m} \quad\left[\begin{array}{ll}
u_{m}, & \overline{\tau_{w} / \mu_{w}}
\end{array}\right]
$$

where $u_{m}$ is still the velocity scale $(\overline{-u})^{2}$. This modification to $u_{T}$ is done to provide better predictions of skin friction for favorable and zero pressure-gradient conditions [14]. The J-K1990A model also takes $A^{+}$ as 17 and replaces equation (9) with the B-L expression (equation (4)), which is modified by the factor $\sigma$ :

$$
\mu_{t, o_{(\mathrm{J}-\mathrm{K}}}=(0.0168)(1.6) \rho F_{\text {wake }} \Gamma_{(\mathrm{B}-\mathrm{L})} \sigma
$$

By using this outer model, Abid et al. avoid the need to find $\delta$. The turbulent diffusion term (equation (14)) is also modified to contribute to the rate equation only in regions of flow recovery (regions where $\sigma \quad 1$ ):

$$
D_{m}=\frac{0.5(\overline{-} m)^{2} \mathrm{~m} \quad\left(\sigma^{2}-1,0 \mathrm{k}\right.}{0.25\left(0 . \delta-y_{m}\right)}
$$

The dissipation length scale is taken to be $m=$ $\min \left(0.4 y_{m}, 0.0 \delta\right)$.

The J-K1990A model was used in reference [13] to predict 3-D separated flow over the ONERA M6 wing successfully on a grid size of $28 \quad 65 \quad 4$. Based on a grid refinement study performed with the B-L model, this grid was determined to be fine enough to yield grid-converged solutions. However, the fact that the model predicted the surface pressures accurately was largely fortuitous. First, an error in the original coding of the Johnson-King turbulence model in TLNS3D moved shocks upstream. Second, computations were originally performed with TLNS3D using scalar dissipation. A reduction in the dissipation levels (through the use of matrix dissipation) can have a dramatic effect on a separated-flow solution, even when the results using scalar dissipation appear to be grid converged. Third, recent computations using both CFL3D and the corrected version of TLNS3D with matrix dissipation indicate that the particular ONERA M6 
case in question may, in fact, be unsteady. Some results and further discussion will be given in the results section.

Johnson and Coakley's modification [14] to the JK model (termed J-K1990J) introduces a blending of the inner model between $\mu_{t, i_{(\mathrm{J}-\mathrm{K})}}$ (equation (8)) and an equilibrium-type model. This blending overcomes the tendency for J-K1985 to incorrectly predict shock locations for attached equilibrium flows. The blended inner eddy viscosity is given by

$$
\mu_{t, i_{(}}=\left(1-\mu_{t, i_{(}}+\mu_{t, i_{(\mathrm{J}-\mathrm{K})}}\right.
$$

where

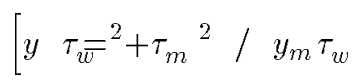

The velocity scale $u_{T}$ is given by equation (18), but the velocity scale $u_{m}$ is modified to include a compressibility correction factor so that

$$
u_{m}=\overline{\rho_{m} / \rho}\left(\overline{-u}{ }_{m}\right)^{2}
$$

The outer model is the same as in J-K1985 (equation (9)); however, a hyperbolic tangent (tanh) blending function is employed to merge the inner and outer models so that

$$
\mu_{t}=\mu_{t, o_{(\mathrm{J}-\mathrm{K})}} \quad\left(\mu_{t, i_{(}} \quad / \mu_{t, o_{(\mathrm{J}-\mathrm{K})}}\right)
$$

This blending is done in place of the exponential (exp) type of blending (equation (17)) to give better predictions of skin friction [14]. The J-K1990J model also takes $A^{+}$as 17 and employs equation (20) for the turbulent diffusion term.

The latest published modification to the J-K model [15] (termed J-K1992) is primarily a new method for determining $\delta$ as a function of $F / F_{m}$, or

$$
\left.\delta=1.2 y \quad{ }_{m}=0.5\right) \quad(
$$

for the Clauser type of outer model (equation (9)). Here, $F$ is the Baldwin-Lomax parameter defined in equation (7), and $m$ denotes maximum. With this expression, the difficulties that are normally associated with computing $\delta$ are avoided. Other than this change, the J-K1992 model is identical to J-K1990J.

In CFL3D and TLNS3D, both the J-K1990A and J-K1992 versions of the Johnson-King turbulence model have been implemented. The Reynolds shear stress $\overline{-u^{\prime} v^{\prime}}(=\tau / \rho)$ is assumed to be given by

$$
\overline{-u^{\prime} v^{\prime}}=\mu_{t} \Omega / \rho
$$

With equation (16), the rate equation (13) is reduced to a time-dependent linear equation that is solved using a multistage explicit Runge-Kutta time-stepping scheme for $g$, as described in reference [13]. The resulting value of $g$ is then combined with the actual value of $\overline{-u^{\prime} v^{\prime}} m$ in the flow field to update $\sigma$ via

$$
\sigma^{t+\Delta t}=\sigma^{t} /\left(\overline{-u^{\prime} v^{\prime}}{ }_{m} g^{2}\right)
$$

The value of $\sigma$ is limited in practice to lie between 0.1 and 4 . In the J-K1992 model, $\mu_{t, i_{(\mathrm{eq})}}$ in equation (21) is taken as $\mu_{t, i(\mathrm{~B}-i),}$ (equation (1)). In wakes, both models revert to the $\mathrm{B}-\mathrm{L}$ methodology. Transition is modeled by setting $\mu_{t}$ to zero along all of the grid lines that are normal to the wall within a preselected range.

The individual effects of the above-mentioned changes and modifications to the $\mathrm{J}-\mathrm{K}$ model on the shock location and extent of separation for 2-D and 3-D separated transonic flows (in the authors' experience) are listed in table 1. In general, the effects of different diffusion-term treatments and of the inner-model blending are relatively small; however, the effects of the other two parameters can be significant, depending on the case. As expected, the compressibility correction factor $\sqrt{\rho_{m} / \rho}$ has little or no effect on transonic solutions. The effects listed in table 1 are more pronounced for more-separated cases; also, 3-D solutions tend to be more sensitive to changes in the model than 2-D solutions.

As discussed in reference [15], the Clauser outer model has a strong experimental foundation. Baldwin and Lomax's original intent was to emulate this expression and avoid the use of $\delta$. However, as shown in reference [15], the B-L outer-model expression can overpredict the eddy-viscosity levels given by the Clauser model by a considerable amount; hence, the B-L outer model, used in combination with the $\mathrm{J}-\mathrm{K}$ inner model (as in J-K1990A), predicts shocks further aft than the Clauser outer model in combination with the J-K inner model (as in J-K1992).

Changing the blending function between the inner and outer models can effect the shock location. Both the exponential (equation (17)) and hyperbolic tangent (equation (24)) blending functions provide a smooth blending; however, the tanh-blended curve lies closer to the original two curves near the point where they cross than the exp-blended curve. Hence, the maximum viscosity values given by the tanh function are slightly higher, and the shock position is generally predicted further downstream.

For the treatment of the turbulent diffusion term, the maximum (max) function was originally introduced 
in references [13] and [14] because $m$ has a negligible effect in regions where $\sigma$ is less than unity. Also, this function generally improved the convergence of the solution over computations that employ the absolute value (abs) function there. Although current computations do not show negligible effects (the shock position is usually altered a small amount), the scheme does benefit from improved convergence when $m$ is employed only in the recovery regions.

Finally, the use of inner-model blending (equation (21)) causes the shock location to move downstream. As an additional significant effect, described in reference [14], the predicted skin-friction levels aft of the shock are reduced and agree better with experimental data and other turbulence models.

Baldwin-Barth. The Baldwin-Barth (B-B) turbulence model [7] is a one-equation turbulence model derived from a simplified form of the - equations. The model solves a partial differential equation (PDE) over the whole field for the modified turbulent Reynolds number :

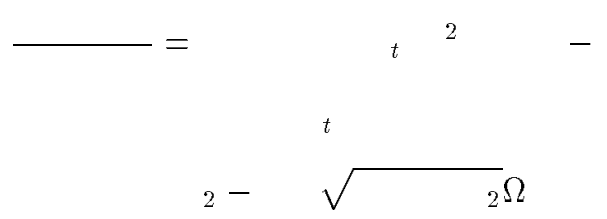

Then

$$
\mu_{t}=\rho \quad 2
$$

The variables $\quad,{ }_{2}$, and ${ }_{2}$ are damping terms that are described in detail in reference [7]. In this formulation of the B-B turbulence model, the thin-layer assumption has been used for the source term (the last term in the PDE).

This equation is solved implicitly using 3-factor AF, with first-order upwind differencing used on the advective terms. Local time-stepping is employed to accelerate convergence. The transition location is modeled by phasing out the source term along all of the grid lines that are normal to the wall within a preselected range.
Spalart-Allmaras. The Spalart-Allmaras (S-A) turbulence model is a one-equation turbulence model derived "using empiricism and arguments of dimensional analysis, Galilean invariance, and selective dependence on the molecular viscosity" [8]. The model solves a PDE over the whole field for a working variable related to the eddy viscosity:

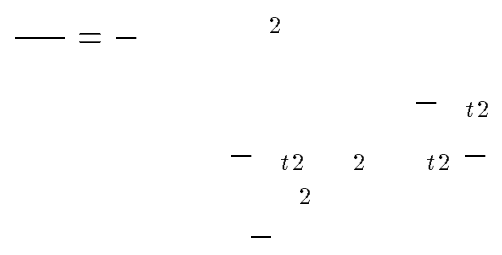

Then

$$
\mu_{t}=\frac{\rho}{/} /
$$

The terms $t 2, \quad 2$, and are described in detail in reference [8]. The term denotes the nearest distance to any wall. The S-A model is very similar in form to the B-B model, although the S-A model includes a destruction term that is not present in the other model. The PDE is solved using the same implicit method used in the B-B model, and transition is modeled in a similar fashion as well.

The B-B and S-A models are relatively new; a consensus of opinion has not yet been reached on their effectiveness in predicting 2-D and 3-D separated aerodynamic flows. Also, from private communication with Barth and Spalart, both of the models are being revised; hence, any conclusions reached in this paper regarding the capabilities of the models are temporary.

Unlike both the B-L and J-K models, the B-B and $\mathrm{S}-\mathrm{A}$ models lend themselves easily to programming on unstructured meshes because no inherent dependency on grid structure exists (e.g., there is no need to locate maximum values of quantities along grid lines normal to the wall). Also, no division into an inner and outer model, or into a wall and wake model, is made. Because they require the solution of a PDE over the whole flow field at each time step, these models are more expensive than B-L or J-K; however, the solution to the PDE need not be converged fully at each time step for iteration toward a steady-state solution. The net result is an increase of less than $20 \%$ in CPU time over the B-L model. 


\section{Results}

\subsection{Airfoil Cases}

All airfoil cases are performed using the 2-D mode of CFL3D with FDS. The cases chosen are case 9 and case 10 from reference [17] for the RAE 2822 airfoil. The wind tunnel corrections for case 9 (attached flow) are essentially those recommended in reference [17]. These corrections are also used by many other researchers, including the authors of references [14] and [18]. The corrections for case 10, taken from reference [7], are slightly different than those recommended in reference [17].

The primary grid used is a $\quad \mathrm{C}$ mesh with 177 points on the airfoil, a minimum spacing at the wall of 0.0000014 (where represents chord), yielding an average + over the body for case 10 of about 0.25 . The outer boundary extent is approximately 15 , and transition is assumed at 3\%.

The conditions used for case 9 are $=$, $=$, and $=$. This case has little to no separation, for which the J-K1985 model predicts the shock location too far upstream [18]. Figures 1(a) and (b) show results computed with J-K1992 and three other turbulence models compared with experimental results. Surface pressure coefficients and upper surface skin-friction coefficients (nondimensionalized by edge values) for all four models are fairly consistent. B$\mathrm{L}$ predicts the shock location the furthest downstream; and $\mathrm{B}-\mathrm{B}$, the furthest upstream.

The conditions used for case 10 are $=$, $=$, and $=$. Figures 2(a) and (b) compare the computed results using the B-L, JK1992, B-B, and S-A models to experimental results. The shock location is computed consistently by the $\mathrm{J}$ K1992, B-B, and S-A models as slightly downstream of the experimental result. The B-L model predicts the shock location even further downstream. Skinfriction coefficients are predicted consistently by the four models upstream of the shock, but significant variation occurs downstream. In particular, the B-B model indicates that the flow does not reattach aft of the shock, unlike the other three models.

These CFL3D solutions are run using a 3-level Wcycle multigrid algorithm. The ${ }_{2}$ norm of the residual of the equation for density generally converges 3 to 4 orders of magnitude in 400 cycles, although the lift generally reaches its steady-state value within 200 to 300 cycles. The CPU times required on the Cray-YMP computer for 400 cycles to obtain the results shown in figure 2 are 214, 230, 249, and $254 \mathrm{CPU}$ seconds for the B-L, J-K1992, B-B, and S-A models, respectively.

The effect of grid refinement on the computed results for the J-K1992 model is shown in figure 3. The coarser grid consists of every other point on the fine grid. The surface pressure predictions do not differ significantly, as shown in figure 3(a). In figure 3(b), the skin friction in front of the shock decreases as the grid is refined, but the change is relatively small between the two grids. These results indicate that the grid is fine enough to adequately remove most grid-related truncation error from the computations. Although not shown, the effect of grid refinement on the results with the other turbulence models is similar.

The differences between the results using J-K1992 and J-K1990A for case 10 are shown in figures 4(a) and (b). The latter model predicts the shock slightly further downstream, in poorer agreement with the experimental results, and predicts skin-friction values that are lower in front of the shock and higher aft of the shock. This comparison in 2-D is shown here because later results in 3-D indicate that the J-K1990A model actually performs better than J-K1992 for a particular separated flow case. The difference between the predicted shock locations with the J-K1992 and J-K1990A models is not as large as the difference reported in reference [15] between the J-K1992 model using the Clauser $\mu_{t}$, versus the $\mathrm{B}-\mathrm{L} \mu_{t}$, . This disagreement is primarily due to the use of the exp blending in J-K1990A (as opposed to tanh blending), which partially counteracts the tendency of the B-L outer model to move shocks downstream. However, the use of exp blending is also the primary cause for the lower skin-friction levels in front of the shock using J-K1990A. The difference in skin friction behind the shock is attributable primarily to the different formulations of the inner model.

\subsection{Wing Cases}

Both CFL3D and TLNS3D are used to compute the flow over the ONERA M6 wing [1] and the Lockheed Wing $C$ [2]. No corrections to the wind tunnel test conditions are employed for the ONERA M6 wing cases, as recommended in reference [1]. For the Lockheed Wing C case, the corrections of Garriz et al. [19] are employed. For most of the ONERA M6 runs, a $\mathrm{C}-\mathrm{O}$ mesh is employed with a minimum normal spacing over the wing of 0.000015 and a distance from the wing to the outer boundary of at least 7.95 . For the Lockheed Wing $\mathrm{C}$ calculations, a $\mathrm{C}-\mathrm{O}$ mesh is employed with a minimum normal spacing over the wing of 0.000001 and a 
distance from the wing to the outer boundary of at least 9.11 . For all computations, transition is taken at approximately $3 \%$.

A more extensive set of computations is performed for the ONERA M6 wing. These include the use of three different test conditions and two different grid densities. The first case computed is an attached flow case $(=, \quad$, and $=$

based on the mean aerodynamic chord). For this case, the minimum normal spacing of the grid yields an average ${ }^{+}$of about 4 over the body. CFL3D results, using FDS, are shown in figures 5(a) through (d) for four different span stations, using four different turbulence models. All models give similar results that are in good agreement with experimental results. Although not shown here, CFL3D with the J-K1990A model and TLNS3D with the B-L, J-K1992, and JK1990A models give nearly identical results.

The first separated-flow case that was attempted is the $=$ case, also reported in reference [13]. Using the J-K1990A model, the solution was found to vary extensively for TLNS3D and CFL3D, depending upon the levels of dissipation added and the grid density. Figure 6(a) shows TLNS3D solutions at span station of a grid with both scalar and matrix dissipation. (Results at other span stations are similar.) The scalar dissipation case converges in excellent agreement with experimental results. However, lowering the dissipation levels gives unsteady, massive separation and moves the shock forward. Although not shown, increasing the grid density to using scalar dissipation causes very little change from the scalar case. This lack of change by itself would suggest a grid-converged solution; however, with the solution using matrix dissipation in mind, this is clearly not the case because solutions using different dissipation levels should tend toward the same steady-state answer as the grid is refined.

CFL3D shows trends similar to TLNS3D, as shown in figure 6(b). Here, the FVS scheme, which has more inherent dissipation than FDS, results in a steady-state solution with the shock position computed in good agreement with experimental results. However, the FDS solution is unsteady and massively separated, with the shock position considerably upstream. These computations indicate that either (1) at these conditions this wing is indeed massively separated and (probably) unsteady, as shown by the computations with low dissipation levels, or (2) a much finer grid is needed to find a grid-converged solution. These computations also demonstrate how easily one can be misled into thinking that a code is giving the "correct' answer, when in fact the truncation error has not been sufficiently reduced through grid-refinement and/or lowering dissipation levels.

Because of questions regarding the nature of the ONERA M6 wing case for = , comparisons are made instead at $=$, for which shock-induced separation exists at a lesser extent. The Mach number for this case is $=$. Results using CFL3D with FDS and four different turbulence models are shown in figures 7(a) and (b) at two representative span stations. As expected, the B-L model predicts the shock too far downstream in the region of the wing where separation exists. The B-B model predicts unsteady, massive separation outboard of about $=$ ahd a shock location too far forward in comparison with experimental data, particularly near the wing tip. Both the J-K1992 and S-A models predict similar shock locations, upstream of experiment at the outboard stations, as well as separation over most of the wing behind the shock at these same stations. TLNS3D solutions with J-K1992 (not shown) show similar behavior.

For the Johnson-King turbulence model, better 3-D results for this case can be obtained using $\mathrm{J}$ K1990A. In 2-D, this version predicts shocks somewhat downstream of the J-K1992 model, and it does not predict skin-friction levels as accurately. (Recall figure 4.) In 3-D, however, the J-K1990A model shows excellent convergence properties and good agreement with experimental surface pressures. It is difficult, therefore, to ignore this model as a viable alternative.

Figures 8(a) through (d) show the CFL3D and TLNS3D results on two different grids for the = case, with the use of the J-K1990A model. As would be expected for grid-converged solutions, results on the two grids for both computer codes are almost the same. Although not shown, TLNS3D results with scalar dissipation and CFL3D results with FVS on the fine grid also give nearly the same results, which provides further evidence that grid convergence has been achieved for this case. The shock is generally predicted slightly downstream of experimental results. Forwardand backward-time particle traces over the wing upper surface for the TLNS3D fine-grid solution, shown in figure 9, clearly delineate the separated regions; flow is from top to bottom.

Convergence properties for CFL3D and TLNS3D for this case on the grid are shown in figures 10(a) and (b). Figure 10(a) shows the of the ${ }_{2}$ norm of the change in density $\rho$ from one cycle to the next. (This method is the standard way that TLNS3D computes residual; CFL3D output has been 
altered to obtain the same information.) Both codes have a similar convergence history, although TLNS3D gives slightly lower residuals at the end of $500 \mathrm{cy}-$ cles. Figure 10(b) shows the lift-coefficient convergence histories. The final for CFL3D is 0.44224 , while TLNS3D gives 0.44225 . CFL3D gets to within $0.5 \%$ of its final lift value in 200 cycles, while TLNS3D achieves the same level in 174 cycles. However, CFL3D (with FDS) runs faster than TLNS3D (with matrix dissipation). On the Cray-YMP computer, 500 cycles with CFL3D requires 4064 seconds; TLNS3D requires 5200 seconds.

The separated-flow Lockheed Wing $\mathrm{C}$ case at the nominal conditions $=, \quad=$, and $=\quad$ is run at the corrected conditions $=,=$, and $=$, as recommended in reference [19]. Computations are performed with CFL3D using several different turbulence models, as well as with TLNS3D using the J-K1990A model. Results are shown in figures 11(a) and (b) at two span stations. At $=$, all furbulence models give roughly the same results, which are in good agreement with experimental results. However, near the wing tip, the B-L model predicts the shock too far aft, and the B-B model predicts it too far forward. All of the other models (including both J-K1992 and J-K1990A) yield consistent results that are in reasonable agreement with experimental results. TLNS3D and CFL3D results with J-K1990A are virtually the same.

\section{Conclusions}

Four turbulence models have been described and evaluated for transonic 2-D and 3-D flows using the computer codes CFL3D and TLNS3D. In particular, different versions of the Johnson-King model have been described and compared. The following observations about all of the turbulence models in general can be made:

1. The Baldwin-Lomax model works well for attached flows; however, shocks are predicted too far downstream for separated flows.

2. The Johnson-King model (version J-K1992), the Baldwin-Barth model, and the Spalart-Allmaras model work well for attached flows and 2-D separated flows, but can predict the shock too far upstream for some 3-D separated flows.

3. Version J-K1990A of the Johnson-King model generally predicts surface pressures for 3-D attached or separated flows very well, but does not work as well as version J-K1992 for 2-D flows (particularly in the prediction of skin friction).

Results of this investigation also indicate that excessive numerical truncation error can lead to an incorrect evaluation of turbulence models. Specifically, the use of scalar dissipation (as opposed to matrix dissipation) in a central-difference scheme, or the use of FVS (as opposed to FDS) in an upwind scheme, can alter the character of 3-D separated-flow solutions. By employing both central-difference and upwind computer codes to a given problem, in addition to performing grid sensitivity studies, this type of uncertainty can be minimized.

\section{References}

[1] Schmitt, V., and Charpin, F., "Pressure Distributions on the ONERA M6 Wing at Transonic Mach Numbers," AGARD AR-138, p. B1, 1979.

[2] Hinson, B., and Burdges, K., "Acquisition and Application of Transonic Wing and Far-Field Test Data for Three-Dimensional Computational Method Evaluation, Volume II - Appendix B, Experimental Data," AFOSR-TR-80-0422, 1980.

[3] Thomas, J., Krist, S., and Anderson, W., "NavierStokes Computations of Vortical Flows Over LowAspect-Ratio Wings," AIAA Journal, Vol. 28, No. 2, pp. 205-212, 1990.

[4] Vatsa, V., and Wedan, B., "Development of a Multigrid Code for 3-D Navier-Stokes Equations and its Application to a Grid-Refinement Study," Computers \& Fluids, Vol. 18, No. 4, pp. 391-403, 1990.

[5] Baldwin, B., and Lomax, H., "Thin Layer Approximation and Algebraic Model for Separated Turbulent Flow," AIAA 78-257, 1978.

[6] Johnson, D., and King, L., "A Mathematically Simple Turbulence Closure Model for Attached and Separated Turbulent Boundary Layers," AIAA Journal, Vol. 23, No. 11, pp. 1684-1692, 1985.

[7] Baldwin, B., and Barth, T., "A One-Equation Turbulent Transport Model for High Reynolds Number Wall-Bounded Flows." NASA TM-102847, 1990.

[8] Spalart, P., and Allmaras, S., "A One-Equation Turbulence Model for Aerodynamic Flows," AIAA 92-0439, 1992.

[9] Roe, P., "Approximate Riemann Solvers, Parameter Vectors, and Difference Schemes," Journal 
of Computational Physics, Vol. 43, pp. 357-372, 1981.

[10] van Leer, B., "Flux Vector Splitting for the Euler Equations," Lecture Notes in Physics, Vol. 170, pp. 501-512, 1982.

[11] Turkel, E., and Vatsa, V., "Effect of Artificial Viscosity on Three-Dimensional Flow Solutions," AIAA 90-1444, 1990.

[12] Johnson, D., "Predictions of Transonic Separated Flow with an Eddy-Viscosity/Reynolds Shear Stress Closure Model," AIAA 85-1683, 1985.

[13] Abid, R., Vatsa, V., Johnson, D., and Wedan, B., "Prediction of Separated Transonic Wing Flows With Nonequilibrium Algebraic Turbulence Model," AIAA Journal, Vol. 28, No. 8, pp. 14261431, 1990.

[14] Johnson, D., and Coakley, T., "Improvements to a Nonequilibrium Algebraic Turbuence Model,"
AIAA Journal, Vol. 28, No. 11, pp. 2000-2003, 1990.

[15] Johnson, D., "Nonequilibrium Algebraic Turbulence Modeling Considerations for Transonic Airfoils and Wings," AIAA 92-0026, 1992.

[16] Holst, T., "Viscous Transonic Airfoil Workshop Compendium of Results," AIAA 87-1460, 1987.

[17] Cook, P., McDonald, M., and Firmin, M., "Airfoil RAE 2822 - Pressure Distributions, and Boundary Layer Wake Measurements," AGARD AR-138, p. A6, 1979.

[18] Coakley, T., "Numerical Simulation of Viscous Transonic Airfoil Flows," AIAA 87-0416, 1987.

[19] Garriz, J., Newman, P., Vatsa, V., Haigler, K., and Burdges, K., "Evaluation of Transonic Wall Interference Assessment and Corrections for SemiSpan Wing Data," AIAA 90-1433, 1990.

\begin{tabular}{|l|l|}
\hline SHOCKS UPSTREAM (MORE SEPARATION) & SHOCKS DOWNSTREAM (LESS SEPARATION) \\
\hline \hline Clauser-type outer model & Baldwin-Lomax-type outer model \\
\hline Exp blending of inner and outer models & Tanh blending of inner and outer models \\
\hline Abs function in diffusion term & Max function in diffusion term \\
\hline J-K inner model & Blended J-K / equilibrium inner model \\
\hline
\end{tabular}

Table 1. Effect of modifications to J-K model. 

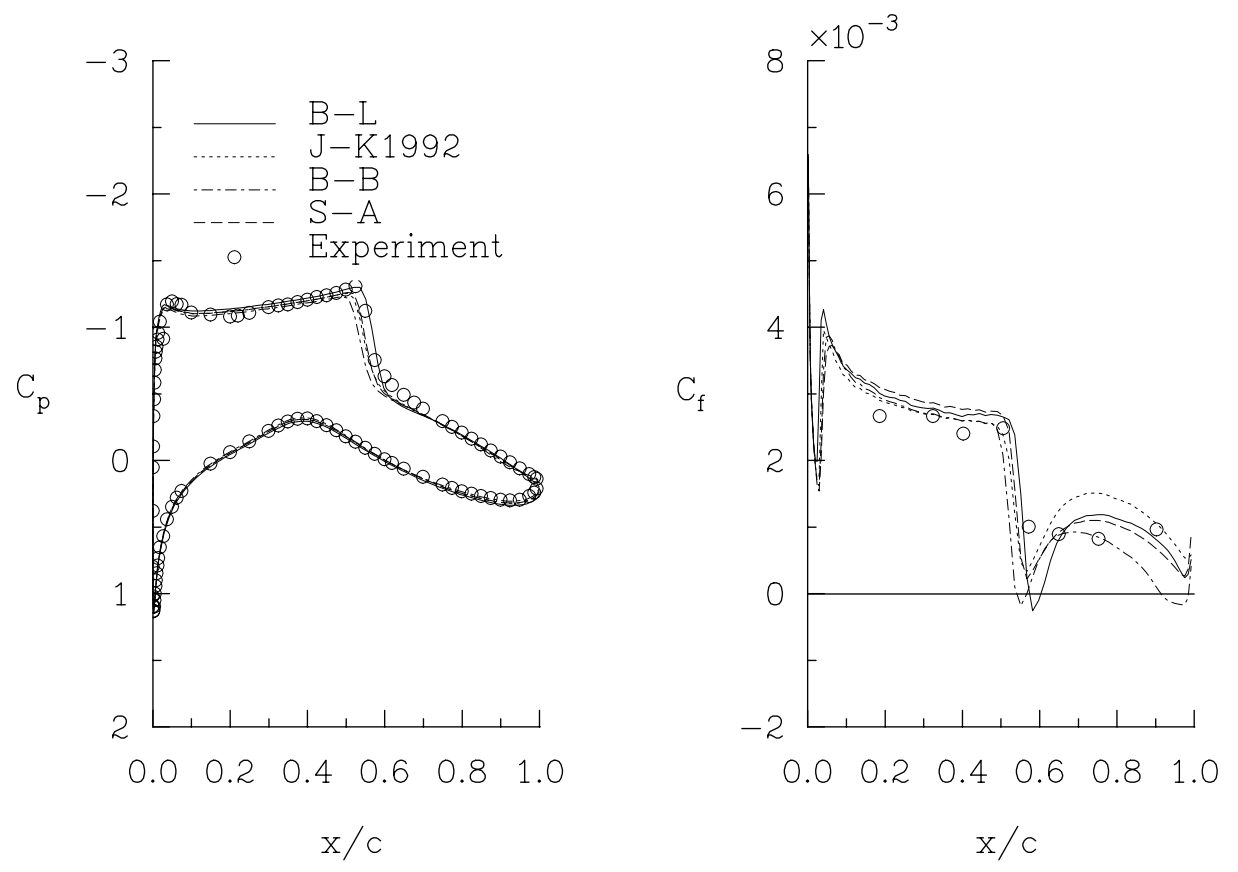

a) surface pressure coefficients

b) skin-friction coefficients

Figure 1. Effect of turbulence model on solution for RAE 2822 airfoil case $9,=,=\quad=\quad, \quad$ grid, CFL3D.

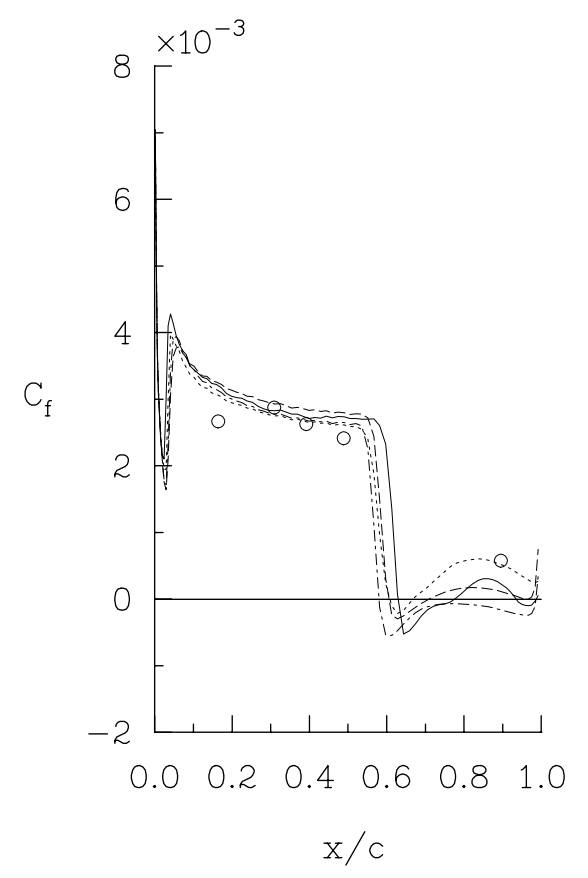

a) surface pressure coefficients

b) skin-friction coefficients

Figure 2. Effect of turbulence model on solution for RAE 2822 airfoil case $10,=,=, \quad, \quad$ grid, CFL3D. 

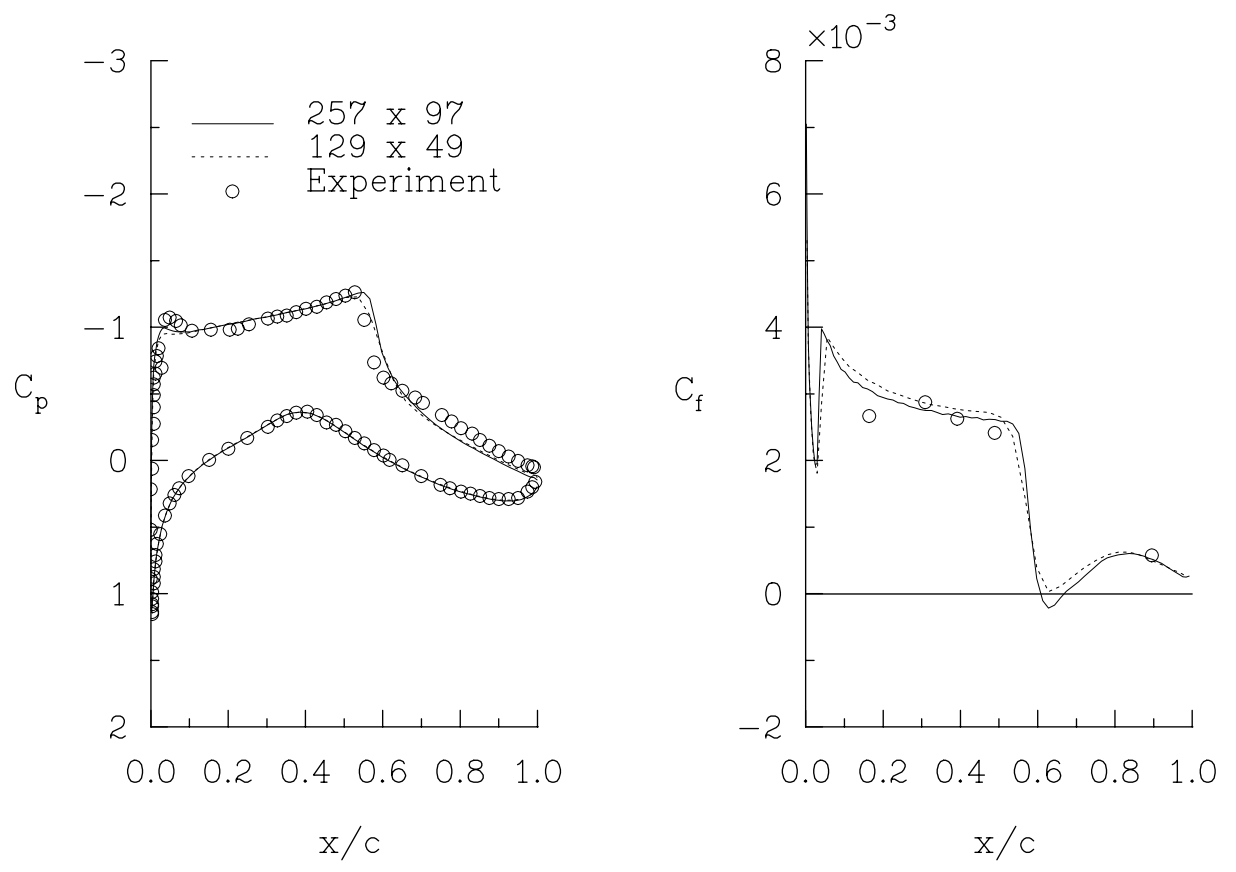

a) surface pressure coefficients

b) skin-friction coefficients

Figure 3. Effect of grid density on solution for case 10, J-K1992 model, CFL3D.

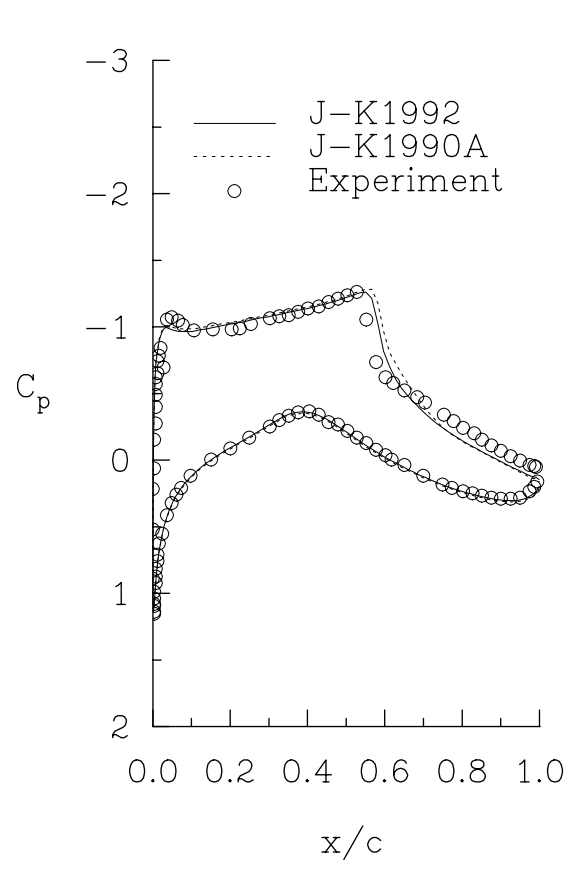

a) surface pressure coefficients

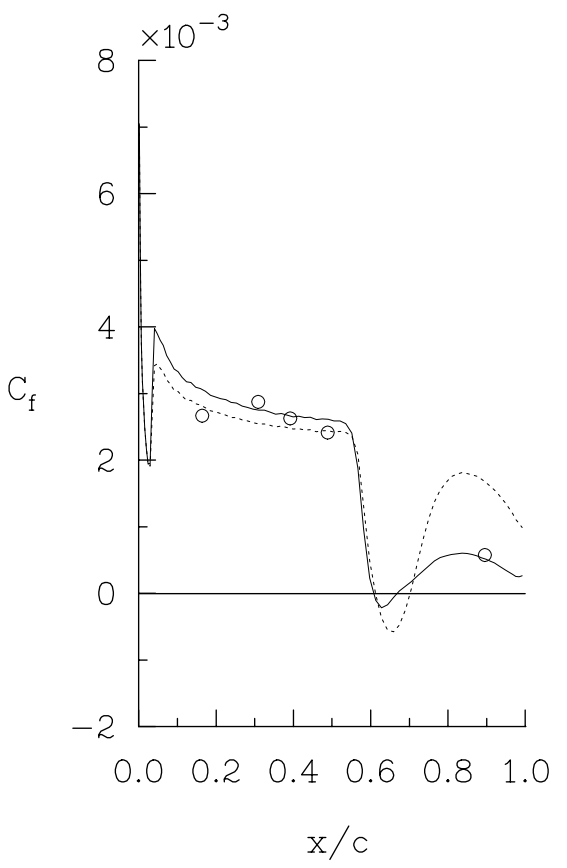

b) skin-friction coefficients

Figure 4. Comparison between J-K1992 and J-K1990A models for case 10, grid, CFL3D. 

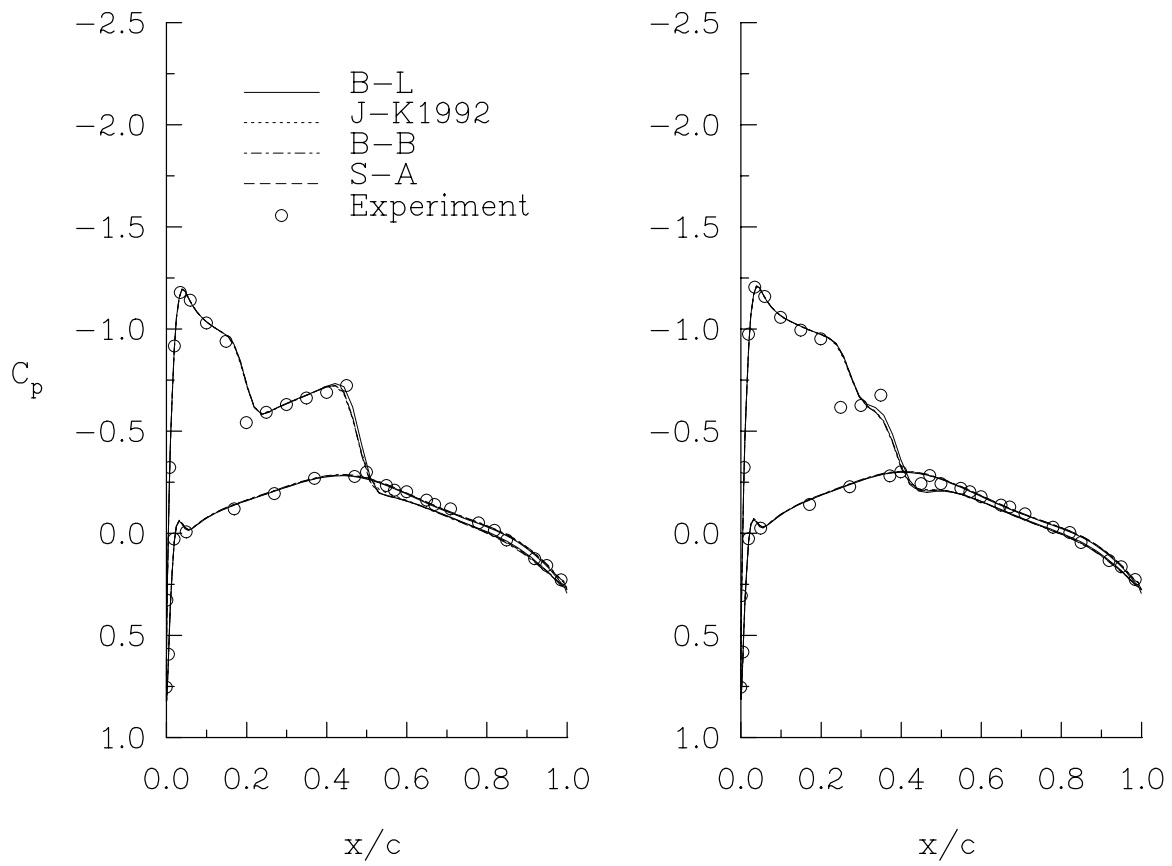

a) $2 \mathrm{y} / \mathrm{B}=0.65$

b) $2 \mathrm{y} / \mathrm{B}=0.80$
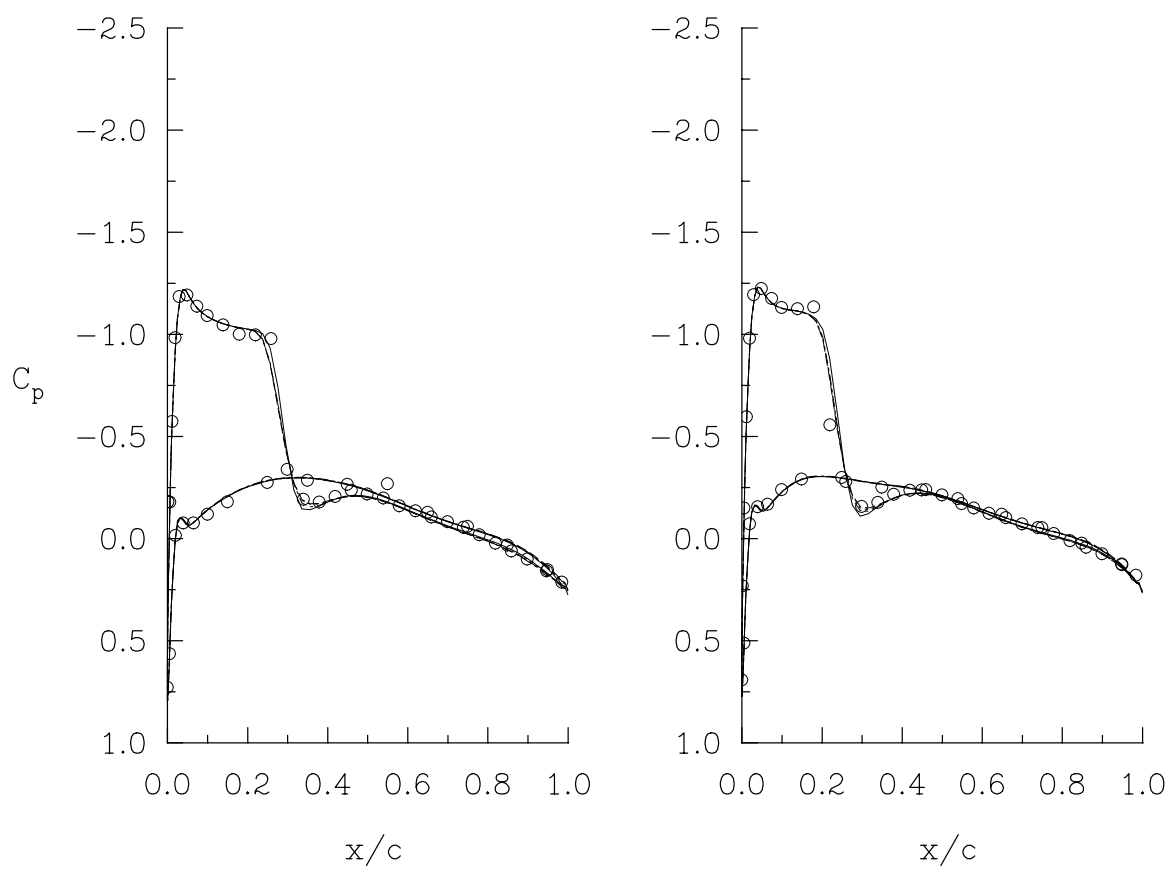

c) $2 \mathrm{y} / \mathrm{B}=0.90$

d) $2 \mathrm{y} / \mathrm{B}=0.95$

Figure 5. Effect of turbulence model on surface pressure coefficients for ONERA M6 wing, $=, \quad=\quad, \quad$ grid, CFL3D. 

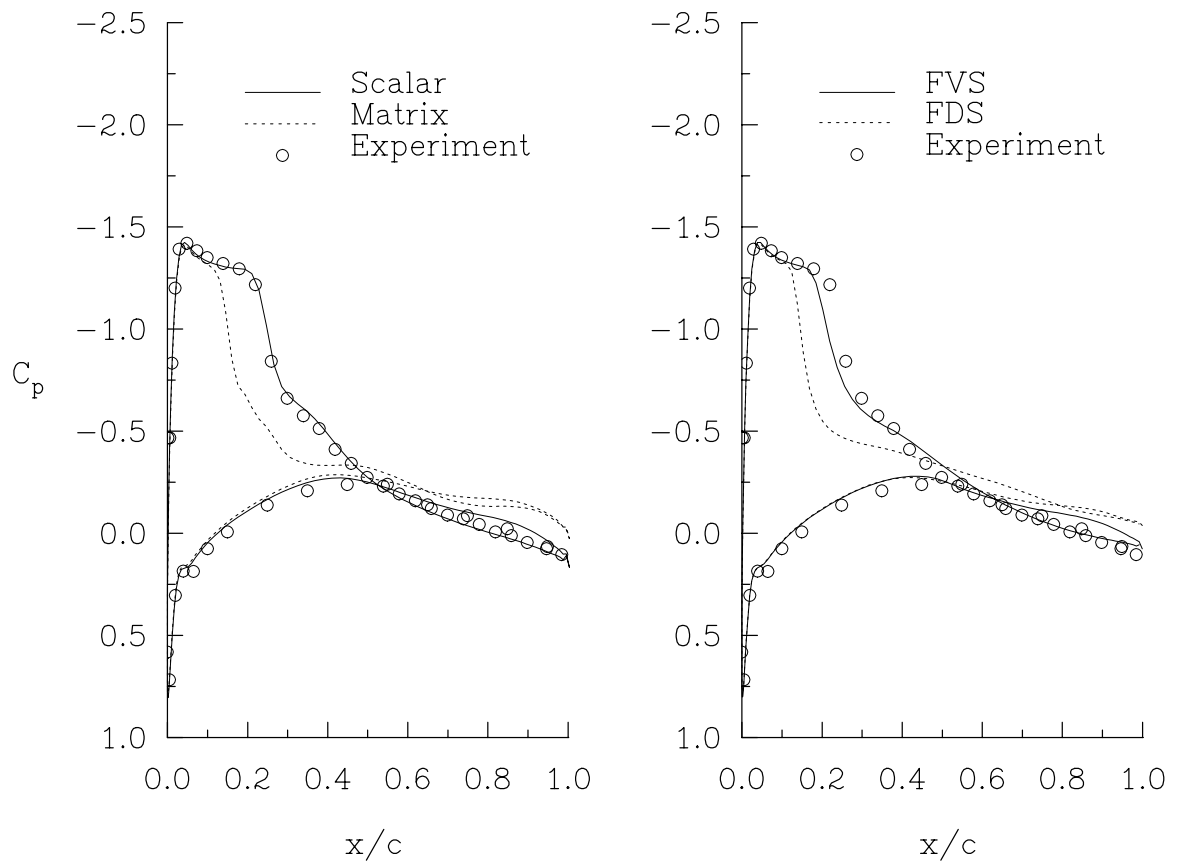

a) TLNS3D

b) CFL3D

Figure 6. Effect of dissipation levels on surface pressure coefficients for ONERA M6 wing, = ,
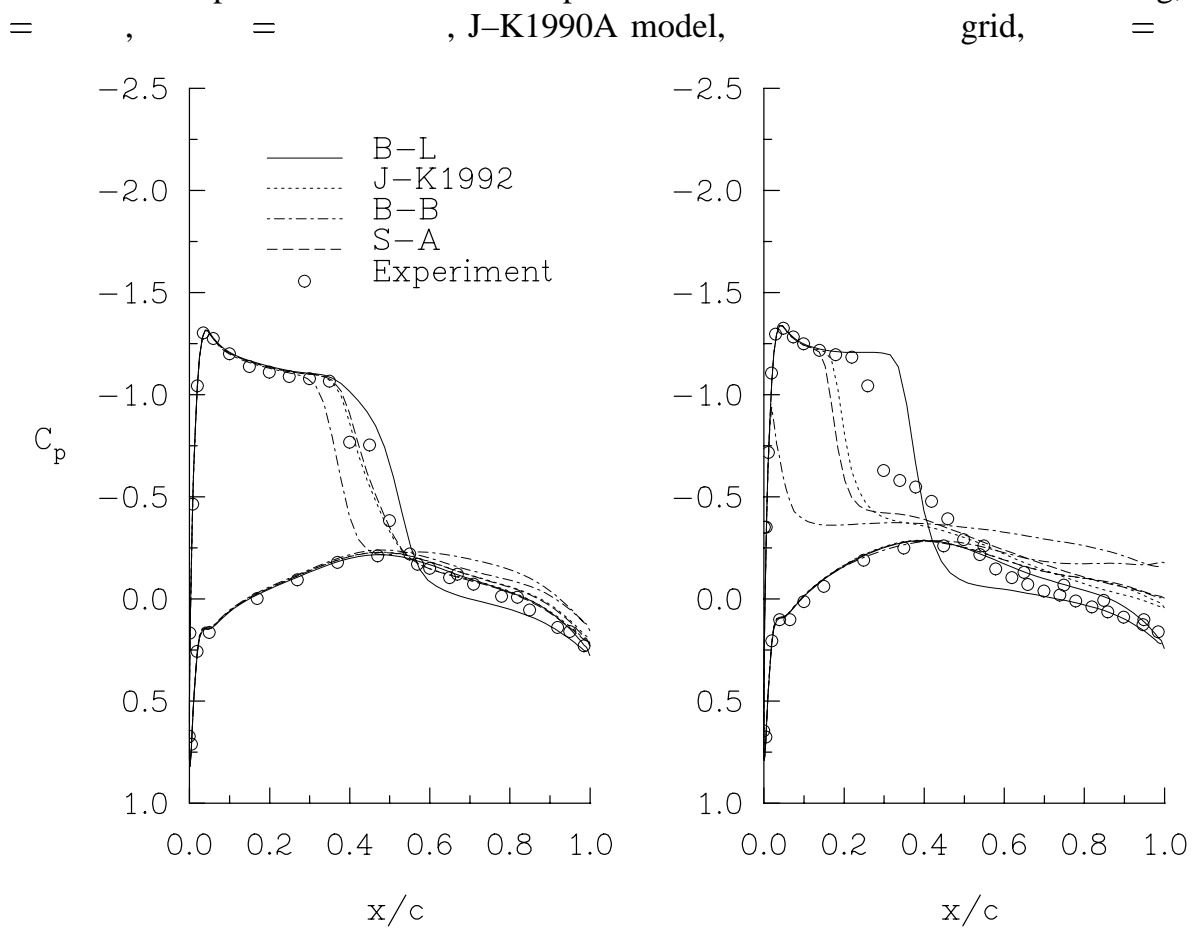
a) $2 \mathrm{y} / \mathrm{B}=0.65$
b) $2 \mathrm{y} / \mathrm{B}=0.90$

Figure 7. Effect of turbulence model on surface pressure coefficients for ONERA M6 wing, $=,=\quad=\quad, \quad$ grid, CFL3D. 

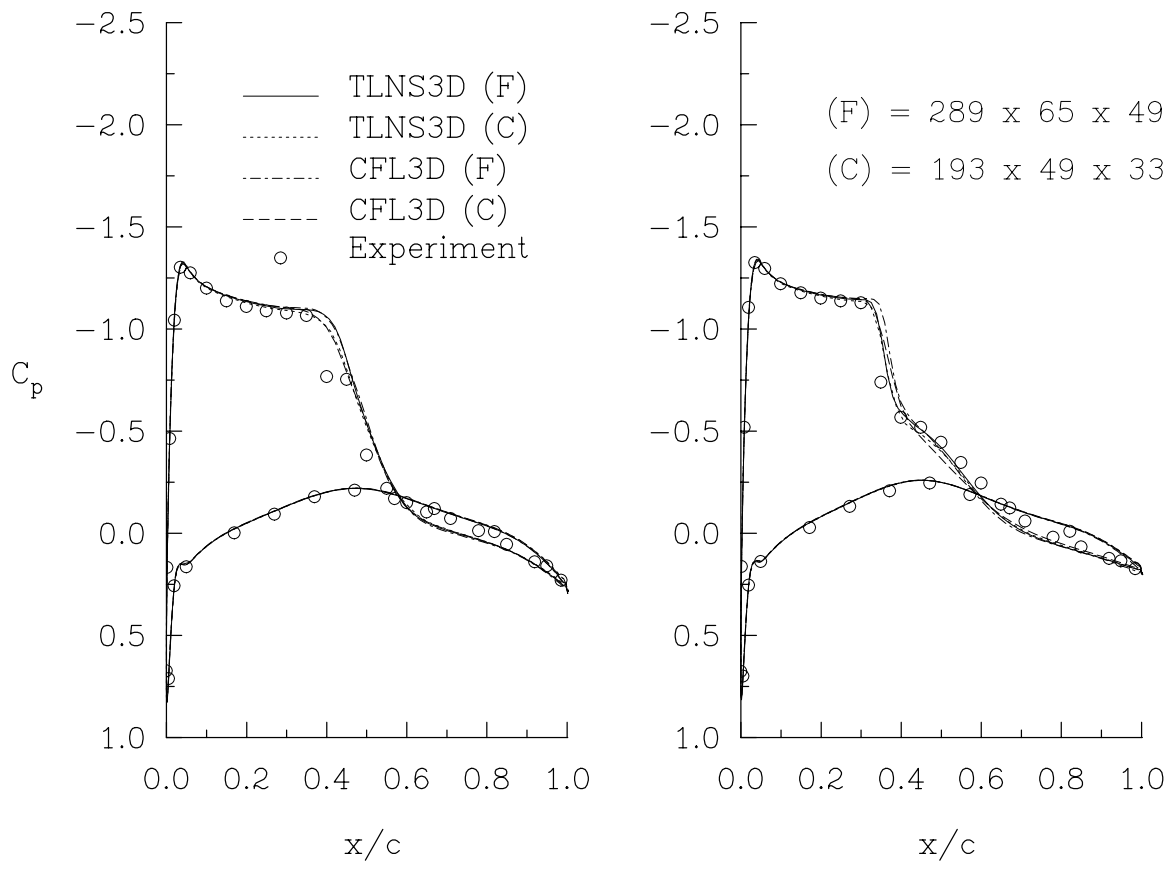

a) $2 y / B=0.65$

b) $2 \mathrm{y} / \mathrm{B}=0.80$
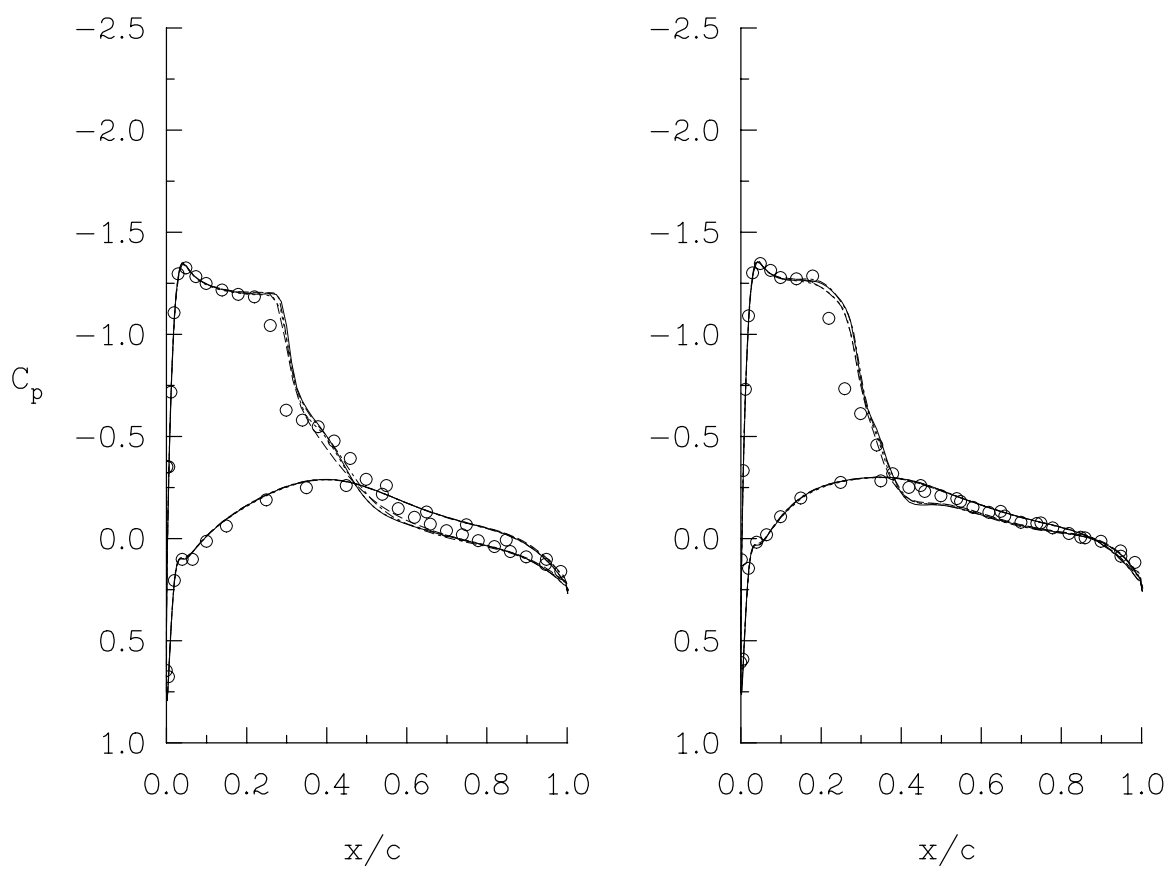

c) $2 y / B=0.90$

d) $2 y / B=0.95$

Figure 8. Effect of grid and computer code on surface pressure coefficients for ONERA M6 wing,
$=\quad, \quad=$, $=$ , J-K1990A model.

$=$ 


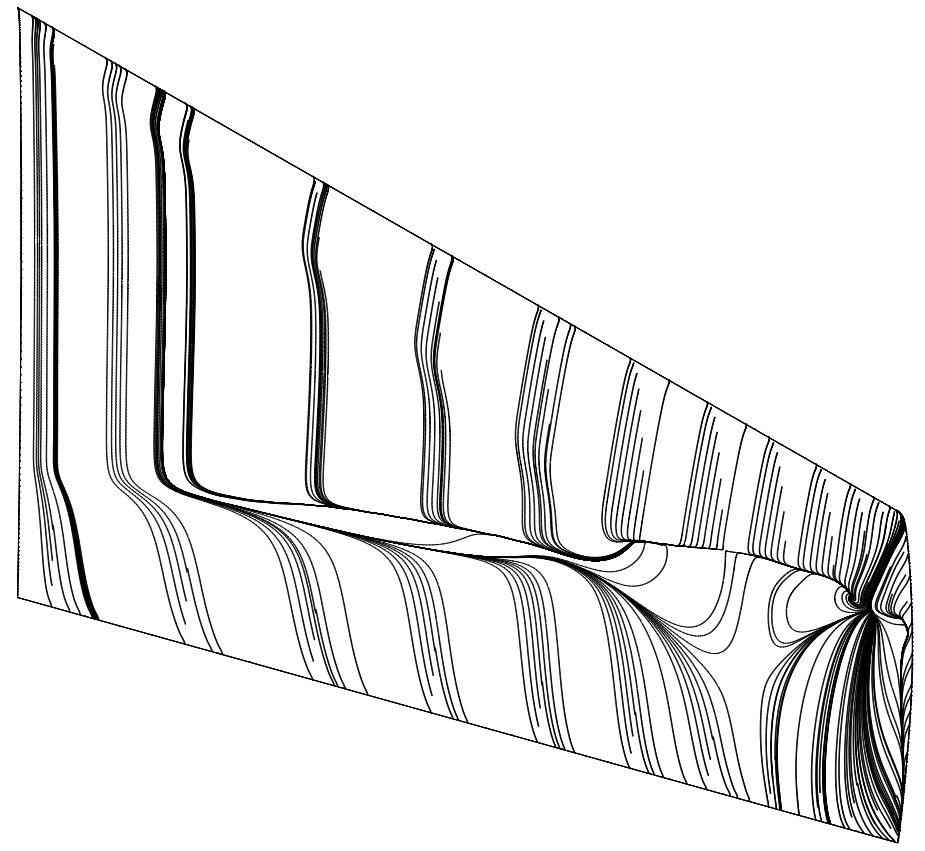

Figure 9. Particle traces over upper surface of ONERA M6 wing, =

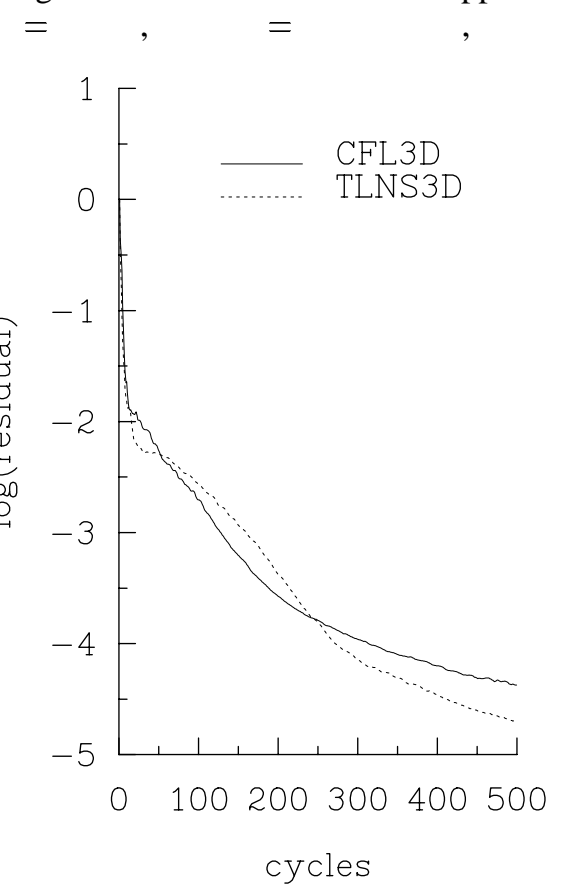

a) residual histories grid, J-K1990A model, TLNS3D.

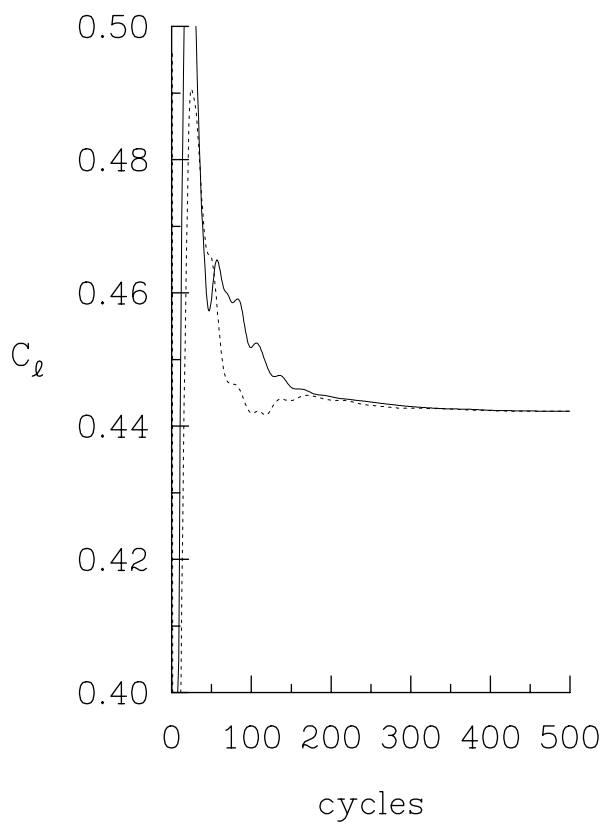

b) lift coefficient histories

Figure 10. Convergence histories for ONERA M6 wing, =

$=\quad, \quad, \quad$ grid, J-K1990A model. 

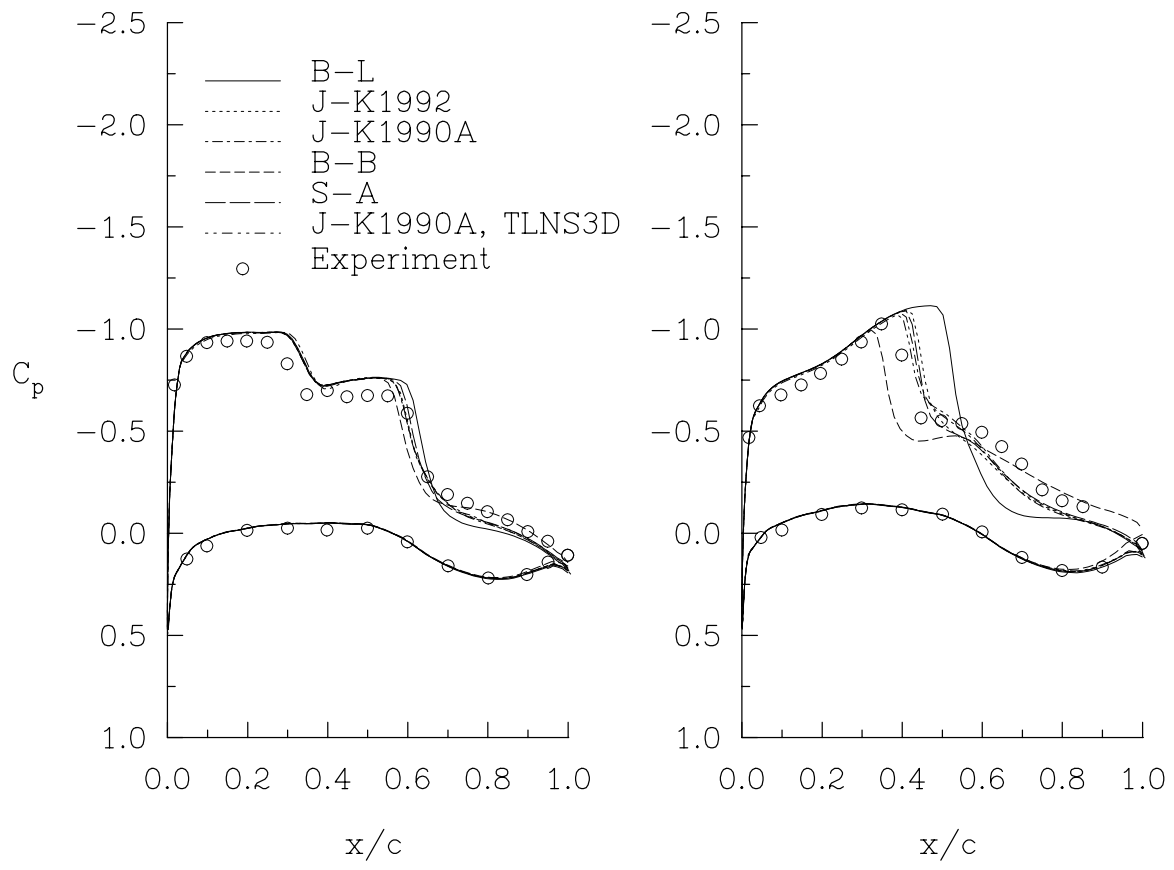
a) $2 \mathrm{y} / \mathrm{B}=0.70$
b) $2 \mathrm{y} / \mathrm{B}=0.90$

Figure 11. Effect of turbulence model on surface pressure coefficients for Lockheed Wing $\mathrm{C}, \quad,=, \quad=\quad, \quad$ grid. 